\title{
Asymmetric Division in the Immuno-Hematopoietic System
}

\author{
Daniel Jimenez-Teja, Nadia Martin-Blanco and Matilde Canelles \\ Instituto de Parasitología y Biomedicina, CSIC, P. T. Ciencias de la Salud, Granada
}

Spain

\section{Introduction}

Asymmetric division is a process by which stem cells asymmetrically segregate certain proteins, called "cell fate determinants", in order to generate two functionally different cells. Normally, one of the daughter cells terminally differentiates while the other retains stem cell properties and continues proliferating. Asymmetric division has been found in virtually all developing systems where stem cells need to simultaneously proliferate and generate differentiated cells: brain, skin, gut, mammary gland, hematopoiesis, also in plants and algae. As a consequence of these studies, it has been established that, by virtue of asymmetric division, both developing and adult organs maintain the delicate equilibrium between proliferation and differentiation. The recent discovery of links to cancer has added momentum to an already very dynamic research area. This review article will discuss the latest developments in the asymmetric division field, with a focus on the immunohematopoietic system.

\section{Historical perspective}

The hypothesis about the existence of asymmetric division was postulated in 1878 based on studies of leech development, where certain cytoplasmic domains of the egg are differentially segregated to the descendants (Whitman, 1878). In the 1980s asymmetric division was described and analyzed in many other organisms, like yeasts, nematode, algae and Drosophila (see Horvitz and Herskowitz 1992 for a comprehensive review). At this stage, it was thought that each organism had a different means to undergo asymmetric division. Daughter cells acquired the differences that made them differentiate into various lineages either intrinsically (by differential inheritance of cytoplasmic or chromosomal factors) or extrinsically, by differential segregation of soluble factors. Intrinsic differences were described in expression of transcription factors, chromatin components, nucleases, receptors, cytoskeletal proteins and others; however, at this point it was not clear which of them were involved in generating asymmetry or were subject to asymmetric segregation themselves to influence differentiation. Extrinsic asymmetric cell division seemed to be the result of either direct cell-cell contact, or secretion of soluble factors. We will focus on intrinsic asymmetric division, which has been most widely studied.

In the 1990s, Drosophila asymmetric division was analyzed in detail and visualized by confocal microscopy (Rhyu, Jan, and Jan 1994). Two proteins with antagonistic function, 
Numb and Notch, were pointed out as the main characters in this complicated process. This was followed by the description of asymmetric division in mammalian brain, with a similar mechanism and also involving asymmetric segregation of Numb (Zhong et al. 1996). At this point, Numb function was unknown, however it was discovered that it could bind (and antagonize) the transmembrane receptor Notch. During the following years, efforts in the two main invertebrate model systems, C. elegans and Drosophila, were focused on the mechanisms to set up cell polarity previous to division, spindle positioning and asymmetric localization of cell fate determinants (Betschinger and Knoblich 2004). Studies on vertebrates showed that many of the proteins involved were conserved, and that there may be a general mechanism for asymmetric division, conserved from the most ancient organisms up to our own brain and muscles. These discoveries resulted on a shift of research in the direction of vertebrates and, concretely, mammalians, and soon asymmetric division was first described in the hematopoietic system (Wu et al. 2007; Schroeder 2007). Studies in Drosophila were still ahead, thus the first link between cancer and asymmetric division was discovered in Drosophila earlier than in mammalians (Caussinus and Gonzalez 2005). In the following years, an important role for phosphorylation of cell fate determinants during mitosis was described (Wirtz-Peitz, Nishimura, and Knoblich 2008). Additionally, the mechanisms for asymmetric inheritance of centrioles (mediated by microtubules), DNA and vesicles were discovered [reviewed in (Neumuller and Knoblich 2009)]. It was realized that, although the proteins involved in asymmetric division are conserved, their roles are different in vertebrates. However, Drosophila studies were vey helpful in the case of the link to cancer, and a molecular mechanism involving Numb and p53 was discovered (Colaluca et al. 2008). The challenge for the next decade will be to integrate all this knowledge at the systems level to understand how asymmetric division works in health and disease, with enormous implications for stem cell research.

\section{Molecular mechanisms of asymmetric division}

Although the mechanism of asymmetric cell division has been intensively studied, there is no general model of how it occurs, because the data have been obtained studying different organisms that normally have their own specificity. Besides, different techniques, depending on the field, have been used to obtain the data, making it difficult to discern real differences from those arising as a result of using different techniques. Another problem, even when dealing with a single model system, is that there are data on asymmetric segregation of different proteins and organelles of the cell, but these data are not connected either temporally or mechanistically. A considerable effort to unify this knowledge into a common model has been made by J. Knoblich, who has continually summarized the data from diverse model systems in a series of excellent reviews [specifically (Knoblich 2010) (Neumuller and Knoblich 2009) are of great help in understanding the underlying mechanisms of asymmetric division]. We will first summarize the current knowledge on how different components of the cell are asymmetrically segregated.

Membrane adaptors- The first stage of asymmetric division in Drosophila neuroblasts is polarization of the cell fate determinants Numb (an endocytic adaptor) and Miranda (an adaptor that recruits other proteins to the membrane), as a result of asymmetric phosphorylation by aPKC (Wirtz-Peitz, Nishimura, and Knoblich 2008). If Numb and Miranda are phosphorylated by aPKC, they cannot localize to the membrane and exert their function (Wirtz-Peitz, Nishimura, and Knoblich 2008). During interphase, aPKC is bound to 
PAR6 and Numb to L(2)GL, which allows Numb to be at the membrane. When the cell enters mitosis, Aurora A phosphorylates PAR6, resulting in L(2)GL phosphorylation and decoupling from Numb, allowing the adaptor PAR3 to bind simultaneously to both Numb and aPKC. Numb is then phosphorylated by aPKC and excluded from the membrane as a consequence (Smith et al. 2007; Wirtz-Peitz, Nishimura, and Knoblich 2008). Since aPKC is asymmetrically positioned in a constitutive fashion, this automatically results in asymmetric membrane distribution of Numb. This mechanism seems to be conserved in mammalians.

Vesicular compartments- Both endocytic adaptors (like Numb) and vesicles have been described to segregate asymmetrically both in Drosophila and mammalians (Zhong et al. 1996; Le Borgne and Schweisguth 2003). Most transmembrane receptors are subject to constant internalization, degradation and recycling, and the balance between these defines signaling levels at each moment. It is also known that receptors inside the endosomes do not only undergo degradation, but are also able to signal, sometimes even at a stronger level than on the membrane (Miaczynska and Bar-Sagi, 2010). This indicates that asymmetric segregation of vesicular compartments is a means to enhance signaling by certain receptors in one of the daughter cells at the expense of the other. Interestingly, such asymmetric segregation of vesicles or proteins involved in endocytosis has been shown to exist in the hematopoietic system (Aguado et al. 2010; Giebel and Beckmann 2007).

Microtubules- During telophase, microtubules play a role in spindle orientation and maintenance of Numb and Miranda asymmetric segregation, although the mechanism is not completely understood (Knoblich 2010).

Centrioles- It has been shown that centrioles are asymmetrically segregated in neuroblasts (the old centriole normally remains with the cell retaining progenitor potential) and this may play a role in cell fate determination (Yamashita et al. 2007).

DNA- There is evidence in some model systems of asymmetric DNA segregation, where the "template" DNA strand is retained by the less differentiated cell. This seems to be true for intestinal epithelium (Potten et al. 1978), muscle (Shinin et al. 2006), and neural stem cells (Karpowicz et al. 2005) but not for hair follicle (Sotiropoulou, Candi, and Blanpain 2008) or hematopoietic (Kiel et al. 2007) stem cells. However, it is not clear whether these disparities arise from looking at cells with different specifics in terms of lag between divisions.

Ribosomal components- In Drosophila, the cell that retains stem cell properties has been shown to present both increased size and enhanced protein synthesis. This seems to be related to asymmetric segregation of ribosomes (Neumuller et al. 2008) and other factors involved in protein synthesis (Fichelson et al. 2009). This has not yet been demonstrated in mammalians, but nevertheless is very intriguing and may be the mechanism by which the capacity to keep proliferating is asymmetrically inherited by just one of the precursors during development.

At this point, the data indicate that the main mechanism of asymmetric division consists on asymmetric inheritance of diverse proteins and subcellular structures, which in its turn helps to enhance the difference between the two daughter cells, so that one can retain stem cell capabilities while the other terminally differentiates. In this way, asymmetric segregation of endocytic adaptors, vesicles and microtubules may contribute to differential signaling in the two daughter cells, while differential inheritance of centrioles, DNA and ribosomes may help preserve stem cell capabilities in just one of the cells. 
Two other important aspects that influence asymmetric division are polarization and spindle orientation during cell division. Polarization has been most extensively studied in C. elegans, where a complex formed by the proteins Par-3, Par-6 and aPKC are already polarized during interphase (Suzuki and Ohno 2006). This mechanism is conserved in Drosophila and is involved in all processes that depend on cell polarity. The mentioned complex is located in the apical part of the cell and, in mammalian cells, is combined with Cadherin and mediates adhesion. Thus, when the spindle forms during cell division, its orientation is crucial to determine symmetry or asymmetry. If the spindle is positioned perpendicular to the Par complex, the cell divides asymmetrically, and the daughter cell that inherits the complex remains a stem cell (probably through adhesion to the stem cell niche), while the other daughter cell abandons the cell niche and differentiates. On the contrary, if the spindle axis parts the Par complex, both daughter cells inherit it and the division is symmetric (Knoblich 2010; Fig. 1).
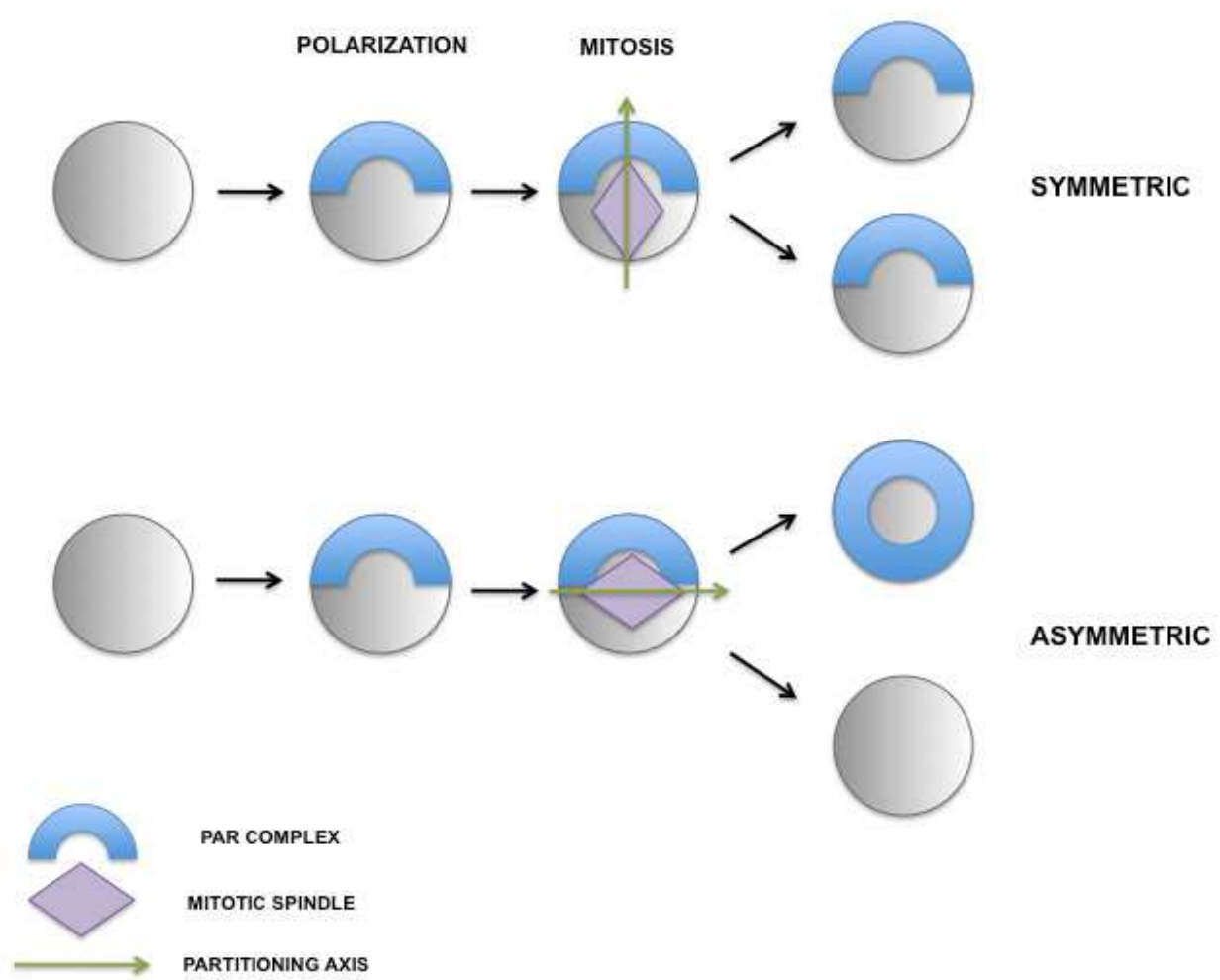

Fig. 1. Polarization and spindle orientation during symmetric or asymmetric division in C. elelgans.

Undoubtedly, in the future all these facts will be unified in a single model explaining how and when things happen during asymmetric division, independently of individual differences among the various model systems used to obtain the data. 


\section{Asymmetric division during normal hematopoiesis}

Hematopoiesis is the process by which about $7 \times 10^{9}$ blood cells are replaced everyday and per $\mathrm{kg}$ to maintain the Hematopoietic Stem Cell (HSC) pool in an organism. On the other hand, a HSC can be defined as a clonogenic cell that has the capacity to self-renew and differentiate into the progenitors of mature blood cells through a symmetric or an asymmetric division, respectively.

The hematopoietic system in mammalians shows a hierarchical structure. There is a wide range of distinct mature cells, such as erythrocytes, megacaryocytes, myeloid cells, mast cells, NK cells, monocytes, B and T cells, and others (Figure 2). All these different cells share a common progenitor cell, the Hematopoietic Stem Cell (HSC). HSCs can divide trough a symmetric process to self-renew or through an asymmetric division process to generate daughter cells with different fates: one daughter cell with the same fate as the progenitor cell, and the second one with Multipotent Progenitor cell fate (MPP). Later, MPPs go downstream through the hierarchy and can divide into three different Oligopotent Progeitors (OPPs). These three different OPPs are Common Lymphoid Progenitors (CLPs), megakaryocyte/erythrocyte progenitors (MEPs) and Common Myeloid Progenitors (CMPs). The last type of OPPs can generate other OPPs such as granulocyte/macrophage progenitors (GMPs) or MEPs. Then, these OPPs derive in a wide range of Lineage Restricted Progenitors, such as pro-B lymphoid cells, pro-T lymphoid cells, pro-NK cells, etc., to finally generate Mature Effector Cells (platelets, dendritic cells, macrophages, erytorocytes, NK, B $\&$ T cells, etc). It must be emphasized that multipotency is lost during this process, therefore, the potency to generate two daughter cells with different fates is reduced from HSCs to mature effector cells (Seita and Weissman 2010).

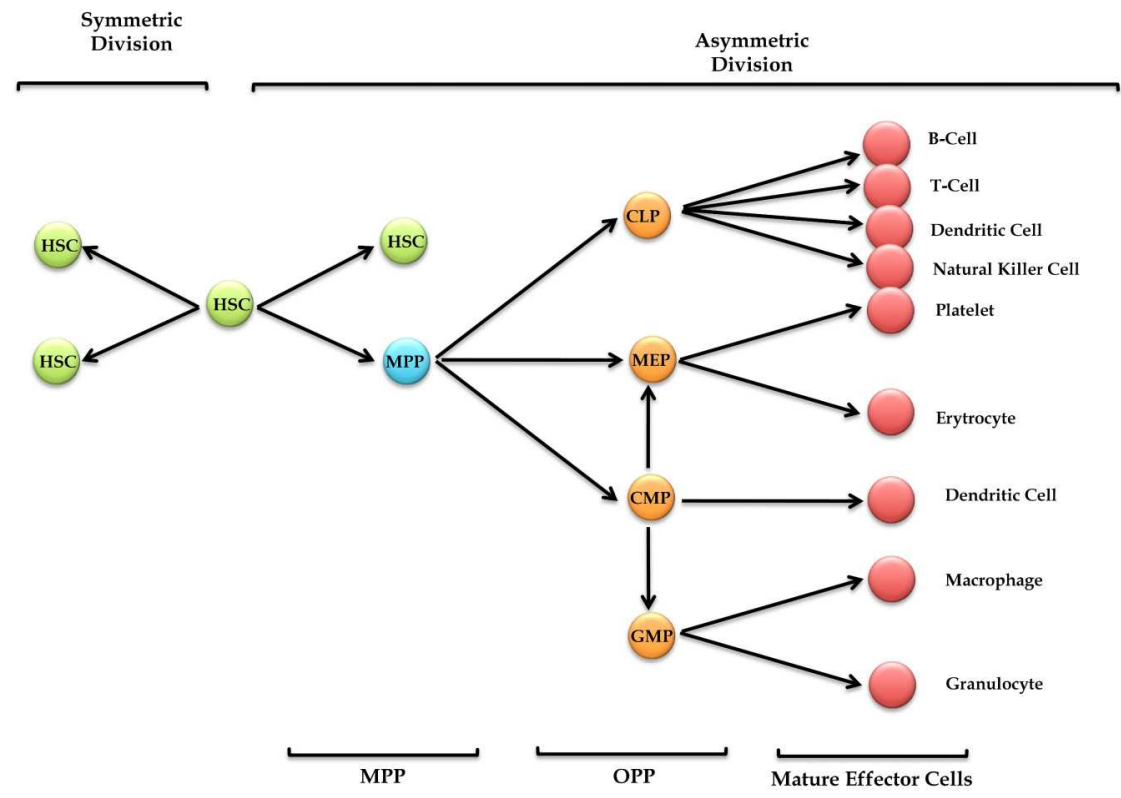

Fig. 2. Schematic representation of precursor decisions during hematopoiesis. 
In symmetric divisions, identical copies of the progenitor cell are generated, maintaining the pool of HSCs. On the other hand, asymmetric divisions contribute to generate diversity. Although it is accepted that the capacity to generate cells with different fates is an intrinsic property of HSCs, and studies with fluorescent proteins have shown that several determinant factors can be asymmetrically distributed during mitosis (Congdon and Reya 2008), the environment has an important role in asymmetric division, as well. Thus, several studies have indicated that the stroma plays an important role in differentiation of HSCs into all blood cell types (Purton and Scadden 2008). In this study, different cell lineages with various fates were obtained culturing the HSCs in the presence of different stromas. Other studies indicate that osteoblasts and endothelial cells act as stem cell niches and may play an important role in progenitor diversity generation. Some experiments show that when HSCs are cultured in the presence of osteoblasts asymmetric division is induced, and symmetric division is more frequent when HSCs are cultured on stromal cells. In addition, experiments where HSCs were cultured in presence or not of Lnk, trombopoeitin (TPO) and several interleukins such as IL-3, IL-6 and IL-11 showed effects in self-renewal and differentiation processes. For instance, Lnk is considered a negative regulator of self-renewal while TPO is a negative regulator of differentiation. In addition, there are other cell types with a potential role as regulators of the HSC niche. One of them is the sympathetic nervous system (Katayama et al. 2006). Therefore, it seems that asymmetric division is an important process for hematopoiesis, although the molecular details remain to be elucidated.

\section{Role of cell fate determinants in hematopoietic malignant proliferation}

The plasma membrane receptor Notch in directly implicated in the proliferative/ differentiative balance of stem cells. Thus, deregulation in Notch signaling is related with several diseases, such as cancer. An increase in Notch signaling results in the development of adenocarcinomas in lung and mammary gland (Allen et al. 2010; Farnie and Clarke 2007). Notch1 can be found in many hematopoietic tissues, such as peripheral $\mathrm{T}$ and $\mathrm{B}$ cells, neutrophils and bone marrow precursors (Stier et al. 2002), and activation of Notch1 increases self-renewal of HSCs while inhibiting the generation of mature cells. This supports previous in vitro studies where Notch activation produced immortalized clones of multipotent cells (Stier et al. 2002; Varnum-Finney et al. 2000), however Notch1 did not completely block the generation of mature cells.

The first proof of the relationship between Notch signaling and cancer was found in acute $\mathrm{T}$ lymphoblastic leukemia (T-ALL), and afterwards Notch signaling was shown to be involved in generation of solid tumors, including melanoma, colorectal cancer, breast cancer, nonsmall cell lung carcinoma and others (Ranganathan, P., et al. 2011). Currently, Notch signaling is receiving increased attention in the development of new therapies against cancer. Some studies have shown that its ligands (specifically Dll4, involved in angiogenesis and $\mathrm{T}$ cell fate specification) are overexpressed in different kinds of cancer (Stylianou S, et al. 2006). As a result, several ways of inhibiting Notch signaling are being tested at different levels:

Synthetic inhibitors. The Notch pathway is inhibited by small compounds, which arrest the proteolysis of Notch receptors by the $\gamma$-secretases-presenilin complex or interfere with the activity of the Notch intracellular domain. The most common $\gamma$-secretases (GSIs) are DAPT and DBZ (dibenzazepine). In addition, specific inhibitors for Dll4-Notch signaling have been 
developed as well. Although different versions of these inhibitors can be found, all of them present the same disadvantage. Initially, these drugs were developed to arrest proteolysis of the amyloid precursor protein (APP) in Alzehimer's disease and therefore, they are not specific and normally interfere with a wide range of different pathways. On the other hand, dnMAML1, a dominant negative of Mastermind-like 1 (MAML1) represents a more selective option. dnMAML1 blocks the transduction of the four known Notch receptors (Notch 1-4). Although dnMAML1 is a potent inhibitor, it shows low levels of cell permeability; for this reason, similar compounds with a better cell permeability have been developed. All these inhibitors down-regulate Notch signaling and have shown good results in treating T-ALL.

Endogenous inhibitors. Endogenous inhibitors, such as Fwb (an E3 ligase), Cbl, Numb and Numblike can be used to regulate Notch signaling by targeting Notch receptors, however, an important disadvantage is their poor specifity. On the other hand, soluble inhibitors such as the extracellular domains of Jagged1, DLk1 and EGFL7 can offer a more specific alternative. However, it must be considered that the mechanism of these inhibitors is not well known and their role in Notch signaling must be studied in detail.

Antibodies. Antibodies against Notch receptors can be used to regulate Notch signaling. Some antibodies have been already developed against Notch1 and Notch3 receptors (Asano N., et al. 2008, Elyaman W., et al 2007, Jurynczyk M., et al 2008, Maekawa Y., et al 2003, Schaller MA., et al. 2007 and Li K., et al. 2008). Antibodies can block specific Notch receptors with a high selectivity, leaving other Notch receptors activated. For example, an anti-Dll4 antibody has been developed against Dll4-Notch signaling and it is showing promising perspectives in anti-angiogenic cancer therapy because of its low toxicity (Ridgway J, et al. 2006). A similar strategy uses molecules called decoys. Decoys are soluble extracellular domains of Notch receptors or ligands. They compete with Notch receptors, inhibiting Notch signaling by binding to endogenous molecules. These associations do not trigger Notch signaling because of lacking the transmembrane region. Notch signaling in endothelial cells has been inhibited using a decoy of Notch1, successfully reducing tumor growth. Other decoys of Dll1, Dll4 and Jagged1 have been successfully developed (Funahashi Y., et al. 2008, Varnum-Finney B., et al. 2000 and Small D., et al. 2001). However, decoys show an important disadvantage. It has been observed that they can be switched from inhibitors into activators easily. The association of decoys with extracellular matrix can produce an activator and trigger Notch proteolysis and activation. The process by which a decoy can be transformed into an activator is not yet fully understood, and this feature makes decoys unpredictable and not valid as therapeutics (Hicks C., et al. 2002).

Notch is regulated by Numb, and loss of this regulation has been described in more than $50 \%$ of human mammary carcinomas. When Numb is lost, Notch signaling is increased, and the balance between self-renewal and differentiation is affected, which results in uncontrolled proliferation. Loss of Numb may be due to ubiquitylation and subsequent proteosomal degradation.

Recent studies carried out by Colaluca et al. (Colaluca et al. 2008) showed that Numb plays an important role in the regulation of the protein p53, also called TP53, an important tumor suppressor involved in $50 \%$ of breast cancers and in $70 \%$ of colon cancers. Numb binds to p53 and the E3 ubiquitin ligase HDM2 (or MDMD2 ligase) to form a triple complex, inhibiting p53 ubiquitylation and, therefore, its degradation. As a consequence, p53 levels are higher and the apparition of breast cancer is diminished. When there is loss of Numb, 
p53 degradation is higher, allowing higher expression of Notch, which results in chemoresistance to the drugs used to combat the disease and in uncontrolled cellular proliferation. Besides, p53 regulates the expression of genes implicated in cell-cycle arrest and apoptosis upon cellular stress. Additionally, it acts as transcriptional factor. Therefore, it seems clear that there is a relationship between Numb deregulation and uncontrolled cellular proliferation via the tumor suppressor p53. However, the mechanism by which Numb regulates p53 remains still unclear (Carter and Vousden 2008).

In some cases, such as in breast cancers, deficiency in Numb expression is due to an increase in ubiquitylation resulting in higher proteasomal degradation. This may be related to increased levels or activity of E3-ligases such as LNK, Siah-1 and MDM2. Another explanation for Numb loss may be ubiquitylation after over-phosphorylation. Restoration of Numb normal levels could be achieved pharmacologically using substances with antiproteasomal activity such as PS-341 or enzymatic inhibitors of Numb degradation (Pece et al. 2004). These investigations have a clear practical application: hopefully, in the future some of the resulting knowledge will be applied to the clinic.

\section{Asymmetric division in the immune system}

During immune system development and function, progenitor cells undergo a series of proliferation and differentiation processes in order to generate the different mature cell populations that protect the body from foreign pathogens. T cells develop in the thymus from bone marrow precursors through a series of intermediate stages. Double negative cells (DN) undergo some division rounds before differentiating into double positive cells (DP), afterwards $\mathrm{T}$ cell progenitors do not divide again in the thymus: only after exiting the thymus and populating the periphery will mature T cells be able to proliferate again. During the immune response, naive $\mathrm{T}$ lymphocytes ( $\mathrm{T}$ lymphocytes recently created that have not encountered antigen) are activated by antigen-presenting cells. Naive T cell activation, through the $\mathrm{T}$ cell receptor (TCR), leads to proliferation and differentiation, triggering a massive expansion of differentiated effector cells, as well as a small number of memory cells (these will remain undifferentiated until subsequent antigen encounters). Thus, after $\mathrm{T}$ cell activation, a single naïve $\mathrm{T}$ cell is able to generate many different $\mathrm{T}$ cell types in order to orchestrate an effective immune response (Stemberger et al. 2007). How can a single cell generate all the $\mathrm{T}$ lymphocyte types that are required for immunity? This question has fascinated immunologists over the past years. Several models have been suggested to explain the generation of subset diversity during the immune response. Some studies suggest a progressive differentiation model (Sallusto, Geginat, and Lanzavecchia 2004), while others suggest an early bifurcation between effector and memory phenotypes, more consistent with asymmetric division, but the question remains controversial. Despite asymmetric division being the most widespread process that regulates the generation of a variety of cell types, this process has only started to be studied in the immune system in the last few years, and it still remains controversial.

Nothing suggests, a priori, that the widespread principle of asymmetric division should not be applied to the thymus, where DN cell proliferation does regulate the total number of cells in the whole organ, and during this process, precursor cells resulting from such divisions must decide between differentiation and proliferation. In this respect, three different aspects should have been studied before making statements about the role of asymmetrically 
segregated cell fate determinants in thymocytes. First, demonstration of the existence of asymmetric division itself (including an assessment of the effect of manipulating asymmetric division); second, identification of cell fate determinants that are asymmetrically segregated and their signaling pathways; finally, elucidation of the mechanisms that lead to asymmetric localization of these determinants, including external cues that regulate cell polarization, as well as intracellular processes that mediate asymmetric segregation of proteins and organelles (as has been described before for studies of both Drosophila and mammalian neural system).

However, the first studies related to asymmetric division in the thymus used either transgenic or knockout mice to over-express or delete Numb (French et al. 2002; Anderson et al. 2005; Wilson et al. 2007). In these studies, investigators used classical assessments of thymocyte differentiation in order to determine whether or not Numb played a role in thymocyte differentiation (they never examined asymmetric division). The conclusion drawn by the three studies was that Numb plays no role in thymus differentiation. However, there are three important considerations that were not taken into account. First, both Numb and its homologue Numblike are expressed in mammalians (the thymus included), and if their levels are reduced so that just $1 \%$ of endogenous levels of either Numb or Numblike remain in the cells, this is still enough to maintain normal asymmetric division (Petersen et al. 2002; Petersen et al. 2006). Second, four different isoforms of Numb are expressed in mammalians (Dho et al. 1999). Third, knockout studies in the immune system must be taken with caution, since there is accumulating evidence that the absence of phenotype does not necessarily mean that the protein does not have a function (Saveliev and Tybulewicz 2009). If Numb acts as a cell fate determinant during asymmetric division in the thymus, one would expect an effect in precursor proliferation rate and the total number of thymocytes, however none of these were examined in these first studies. Nevertheless, the existence of three studies claiming no role for Numb in the thymus predisposed the whole field against the notion of asymmetric division.

Fortunately, over the past few years, the first studies on asymmetric division in the thymus and peripheral $\mathrm{T}$ lymphocytes performed following a more logical order (i.e., examining in the first place asymmetric segregation of determinants) have provided exciting data about asymmetric division in the immune system (Aguado et al. 2010; Chang et al. 2007). In the first study, our group showed by confocal microscopy that Numb is segregated asymmetrically during thymocyte division. By inhibiting Numb (using a dominant negative), or overexpressing it, we showed that functional Numb levels determine DN thymocyte proliferation rate and, ultimately, thymus size. Furthermore, we showed that Numb can regulate pre-TCR localization and signaling, acting as an endocytic protein. As a result, a model was proposed where thymocytes divide by asymmetric division to generate one daughter cell that inherits Numb and keeps precursor properties and a second that does not inherit Numb and receives pre-TCR signaling as a consequence, which results in differentiation (Fig. 3). The second study showed that peripheral $\mathrm{CD}^{+} \mathrm{T}$ cells do indeed undergo asymmetric division, and this process regulates the choice between effector and memory differentiation (Chang et al. 2007). The authors showed that after the first naïve $\mathrm{CD}^{+} \mathrm{T}$ cell division, the proximal and distal daughter cells have different phenotypes. Thus, proximal daughter cells expressed low CD62L levels and higher CD69, CD43 and CD25 levels. Furthermore, when these cells were transferred into naïve secondary recipients, they provided protection against acute infection, but poor long-term protection, a profile 
consistent with the effector lineage. However, distal daughters expressed high levels of CD62L and lower levels of CD69, CD43, CD25 and CD44 and these cells provided a good long-term protection in vivo, a profile more consistent with the central memory cells. This is clear evidence that asymmetric division occurs, at least during the first division.

As we have explained before, for asymmetric division to occur, the progenitor cell needs to receive an external cue to dictate the axis of polarity, recruit cell fate determinants and align the mitotic spindle with a correct position that ensures asymmetric segregation of the determinants. During mature $\mathrm{T}$ cell division, the axis of polarity and mitotic spindle alignment are established by the formation of the immunological synapse. The immunological synapse has been extensively studied as a site of clustered signaling molecules, and can be considered as a marker of the polarized $\mathrm{T}$ cell and a mechanism for asymmetric division regulation. Recent studies showed that asymmetric cell division is not observed either during non antigen-dependent activation or the second and subsequent cell divisions following antigen stimulation, and that the polarity cue for asymmetric cell division requires the contact with antigen-presenting cells (Chang et al. 2007; Oliaro et al. 2010; Fig. 3). One problem with this model is that if it is just the first division that is
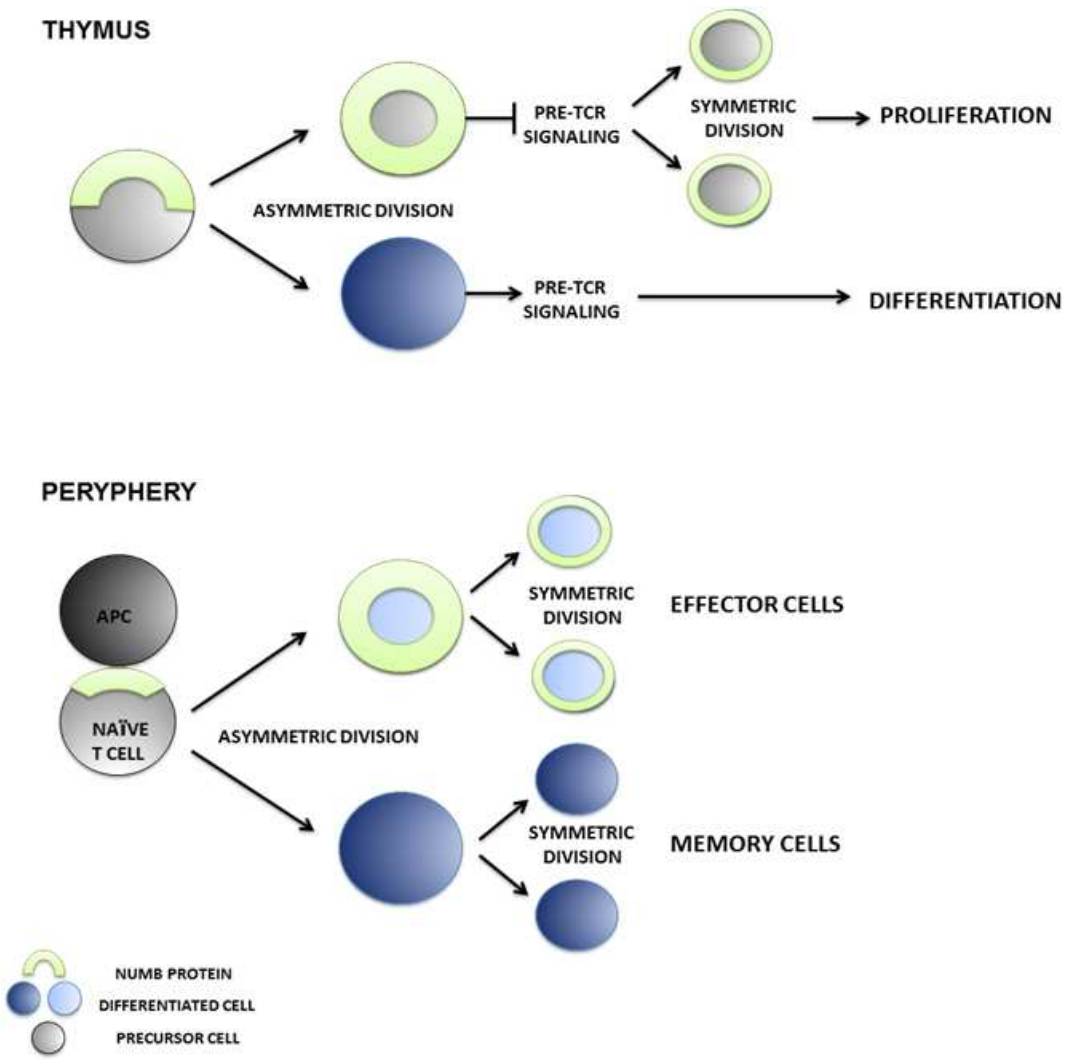

Fig. 3. Schematic representation of the current models for asymmetric division in thymus and periphery. 
asymmetric during the immune response, and all the subsequent divisions are symmetric, it is not clear how the final numbers of memory and effector cells are achieved. In any case, these data on thymus and peripheral $\mathrm{T}$ cells demonstrate that the immunological system is not a remarkable exception to the principle of asymmetric division as the universal mechanism to ensure a correct balance between expansion and differentiation during development. The mechanistic details on how asymmetric division is orchestrated in the immune system in order to achieve correct numbers of mature cells will surely be elucidated soon.

\section{Future directions of the field}

Asymmetric division has transitioned from being an intriguing but unexplained anomaly of neural development into a fertile field where scientists working on different developmental biology areas converge to exchange methods and ideas. The recently discovered link to cancer stresses out the importance of these studies in the immuno-hematopoietic system.

An important current challenge for the field of asymmetric division is unification of knowledge. A general model for the functioning of asymmetric division that applies to all organisms and tissues needs to be postulated, even if it is very schematic at the beginning. Next, unification of methods should be achieved: the same phenomenon in different organisms should not be studied using different techniques simply because researchers of different areas feel more comfortable with a certain approach. To avoid this, more joint scientific meetings on asymmetric division must be organized, so that researchers can exchange views and knowledge, besides funding should be available for those willing to assume the risk of applying new techniques to old model systems. If the field does not evolve in this way, it risks loosing its current novelty and drive. Hopefully, new exciting discoveries will keep the area alive, and the many open questions about how organisms and tissues orchestrate growth and differentiation will be answered soon.

\section{References}

Aguado, R., N. Martin-Blanco, M. Caraballo, and M. Canelles. 2010. The endocytic adaptor Numb regulates thymus size by modulating pre-TCR signaling during asymmetric division. Blood 116 (10):1705-14.

Allen, T. D., E. M. Rodriguez, K. D. Jones, and J. M. Bishop. 2010. Activated notch1 induces lung adenomas in mice and cooperates with myc in the generation of lung adenocarcinoma. Cancer Res 71 (18):6010-8.

Anderson, A. C., E. A. Kitchens, S. W. Chan, C. St Hill, Y. N. Jan, W. Zhong, and E. A. Robey. 2005. The Notch regulator Numb links the Notch and TCR signaling pathways. J Immunol 174 (2):890-7.

Asano N., Watanabe T., Kitani A., et al. 2008. Notch1 signaling and regulatory $\mathrm{T}$ cell function. J Immunol 180: 2796-804.

Betschinger, J., and J. A. Knoblich. 2004. Dare to be different: asymmetric cell division in Drosophila, C. elegans and vertebrates. Curr Biol 14 (16):R674-85.

Carter, S., and K. H. Vousden. 2008. A role for Numb in p53 stabilization. Genome Biol 9 (5):221. 
Caussinus, E., and C. Gonzalez. 2005. Induction of tumor growth by altered stem-cell asymmetric division in Drosophila melanogaster. Nat Genet 37 (10):1125-9.

Chang, J. T., V. R. Palanivel, I. Kinjyo, F. Schambach, A. M. Intlekofer, A. Banerjee, S. A. Longworth, K. E. Vinup, P. Mrass, J. Oliaro, N. Killeen, J. S. Orange, S. M. Russell, W. Weninger, and S. L. Reiner. 2007. Asymmetric T lymphocyte division in the initiation of adaptive immune responses. Science 315 (5819):1687-91.

Colaluca, I. N., D. Tosoni, P. Nuciforo, F. Senic-Matuglia, V. Galimberti, G. Viale, S. Pece, and P. P. Di Fiore. 2008. NUMB controls p53 tumour suppressor activity. Nature 451 (7174):76-80.

Congdon, K. L., and T. Reya. 2008. Divide and conquer: how asymmetric division shapes cell fate in the hematopoietic system. Curr Opin Immunol 20 (3):302-7.

Dho, S. E., M. B. French, S. A. Woods, and C. J. McGlade. 1999. Characterization of four mammalian numb protein isoforms. Identification of cytoplasmic and membraneassociated variants of the phosphotyrosine binding domain. J Biol Chem 274 (46):33097-104.

Elyaman W., Bradshaw E. M., Wang Y., et al. 2007. JAGGED1 and delta1 differentially regulate the outcome of experimental autoimmune encephalomyelitis. J Immunol 179: 5990-98.

Farnie, G., and R. B. Clarke. 2007. Mammary stem cells and breast cancer--role of Notch signalling. Stem Cell Rev 3 (2):169-75.

Fichelson, P., C. Moch, K. Ivanovitch, C. Martin, C. M. Sidor, J. A. Lepesant, Y. Bellaiche, and J. R. Huynh. 2009. Live-imaging of single stem cells within their niche reveals that a U3snoRNP component segregates asymmetrically and is required for selfrenewal in Drosophila. Nat Cell Biol 11 (6):685-93.

French, M. B., U. Koch, R. E. Shaye, M. A. McGill, S. E. Dho, C. J. Guidos, and C. J. McGlade. 2002. Transgenic expression of numb inhibits notch signaling in immature thymocytes but does not alter T cell fate specification. J Immunol 168 (7):3173-80.

Funahashi Y., Hernandez S. L., Das I., et al. 2008. A notch1 ectodomain construct inhibits endothelial notch signaling, tumor growth, and angiogenesis. Cancer Res 68: 4727-35.

Giebel, B., and J. Beckmann. 2007. Asymmetric cell divisions of human hematopoietic stem and progenitor cells meet endosomes. Cell Cycle 6 (18):2201-4.

Hicks C., Ladi E., Lindsell C., et al. 2002. A secreted Delta1-Fc fusion protein functions both as an activator and inhibitor of Notch1 signaling. J Neurosci Res 68: 655-67.

Horvitz, H. R., and I. Herskowitz. 1992. Mechanisms of asymmetric cell division: two Bs or not two Bs, that is the question. Cell 68 (2):237-55.

Jurynczyk M., Jurewicz A., Raine C. S., et al. 2008. Notch3 inhibition in myelin-reactive T cells down-regulates protein kinase $C$ theta and attenuates experimental autoimmune encephalomyelitis. J Immunol 180: 2634- 40.

Karpowicz, P., C. Morshead, A. Kam, E. Jervis, J. Ramunas, V. Cheng, and D. van der Kooy. 2005. Support for the immortal strand hypothesis: neural stem cells partition DNA asymmetrically in vitro. J Cell Biol 170 (5):721-32.

Katayama, Y., M. Battista, W. M. Kao, A. Hidalgo, A. J. Peired, S. A. Thomas, and P. S. Frenette. 2006. Signals from the sympathetic nervous system regulate hematopoietic stem cell egress from bone marrow. Cell 124 (2):407-21. 
Kiel, M. J., S. He, R. Ashkenazi, S. N. Gentry, M. Teta, J. A. Kushner, T. L. Jackson, and S. J. Morrison. 2007. Haematopoietic stem cells do not asymmetrically segregate chromosomes or retain BrdU. Nature 449 (7159):238-42.

Knoblich, J. A. 2010. Asymmetric cell division: recent developments and their implications for tumour biology. Nat Rev Mol Cell Biol 11 (12):849-60.

Le Borgne, R., and F. Schweisguth. 2003. Unequal segregation of Neuralized biases Notch activation during asymmetric cell division. Dev Cell 5 (1):139-48.

Li K., Li Y., Wu W., et al. 2008. Modulation of Notch signaling by antibodies specific for the extracellular negative regulatory region of NOTCH3. J Biol Chem 283: 8046-54.

Maekawa Y., Tsukumo S., Chiba S., et al. 2003. Delta1-Notch3 inter- actions bias the functional differentiation of activated CD4p T cells. Immunity 19: 549-59.

Miaczynska, M., and D. Bar-Sagi. 2010. Signaling endosomes: seeing is believing. Curr Opin Cell Biol 22 (4):535-40.

Neumuller, R. A., and J. A. Knoblich. 2009. Dividing cellular asymmetry: asymmetric cell division and its implications for stem cells and cancer. Genes Dev 23 (23):2675-99.

Neumuller, R. A., J. Betschinger, A. Fischer, N. Bushati, I. Poernbacher, K. Mechtler, S. M. Cohen, and J. A. Knoblich. 2008. Mei-P26 regulates microRNAs and cell growth in the Drosophila ovarian stem cell lineage. Nature 454 (7201):241-5.

Oliaro, J., V. Van Ham, F. Sacirbegovic, A. Pasam, Z. Bomzon, K. Pham, M. J. LudfordMenting, N. J. Waterhouse, M. Bots, E. D. Hawkins, S. V. Watt, L. A. Cluse, C. J. Clarke, D. J. Izon, J. T. Chang, N. Thompson, M. Gu, R. W. Johnstone, M. J. Smyth, P. O. Humbert, S. L. Reiner, and S. M. Russell. 2010. Asymmetric cell division of T cells upon antigen presentation uses multiple conserved mechanisms. J Immunol 185 (1):367-75.

Pece, S., M. Serresi, E. Santolini, M. Capra, E. Hulleman, V. Galimberti, S. Zurrida, P. Maisonneuve, G. Viale, and P. P. Di Fiore. 2004. Loss of negative regulation by Numb over Notch is relevant to human breast carcinogenesis. J Cell Biol 167 (2):215-21.

Petersen, P. H., H. Tang, K. Zou, and W. Zhong. 2006. The enigma of the numb-Notch relationship during mammalian embryogenesis. Dev Neurosci 28 (1-2):156-68.

Petersen, P. H., K. Zou, J. K. Hwang, Y. N. Jan, and W. Zhong. 2002. Progenitor cell maintenance requires numb and numblike during mouse neurogenesis. Nature 419 (6910):929-34.

Potten, C. S., W. J. Hume, P. Reid, and J. Cairns. 1978. The segregation of DNA in epithelial stem cells. Cell 15 (3):899-906.

Purton, L. E., and D. T. Scadden. 2008. The hematopoietic stem cell niche.

Rhyu, M. S., L. Y. Jan, and Y. N. Jan. 1994. Asymmetric distribution of numb protein during division of the sensory organ precursor cell confers distinct fates to daughter cells. Cell 76 (3):477-91.

Sallusto, F., J. Geginat, and A. Lanzavecchia. 2004. Central memory and effector memory T cell subsets: function, generation, and maintenance. Annu Rev Immunol 22:745-63.

Saveliev, A., and V. L. Tybulewicz. 2009. Lymphocyte signaling: beyond knockouts. Nat Immunol 10 (4):361-4.

Schaller M. A., Neupane R., Rudd B. D., et al. 2007. Notch ligand Delta-like 4 regulates disease pathogenesis during respiratory viral infections by modulating Th2 cytokines. J Exp Med 204: 2925-34. 
Schroeder, T. 2007. Asymmetric Cell Division in Normal and Malignant Hematopoietic Precursor Cells. Cell Stem Cell 1 (5):479-481.

Seita, J., and I. L. Weissman. 2010. Hematopoietic stem cell: self-renewal versus differentiation. Wiley Interdiscip Rev Syst Biol Med 2 (6):640-53.

Shinin, V., B. Gayraud-Morel, D. Gomes, and S. Tajbakhsh. 2006. Asymmetric division and cosegregation of template DNA strands in adult muscle satellite cells. Nat Cell Biol 8 (7):677-87.

Small D., Kovalenko D., Kacer D., et al. 2001. Soluble Jagged 1 represses the function of its transmembrane form to induce the formation of the Src- dependent chord-like phenotype. J Biol Chem 276: 32022-30.

Smith, C. A., K. M. Lau, Z. Rahmani, S. E. Dho, G. Brothers, Y. M. She, D. M. Berry, E. Bonneil, P. Thibault, F. Schweisguth, R. Le Borgne, and C. J. McGlade. 2007. aPKCmediated phosphorylation regulates asymmetric membrane localization of the cell fate determinant Numb. EMBO J 26 (2):468-80.

Sotiropoulou, P. A., A. Candi, and C. Blanpain. 2008. The majority of multipotent epidermal stem cells do not protect their genome by asymmetrical chromosome segregation. Stem Cells 26 (11):2964-73.

Stemberger, C., K. M. Huster, M. Koffler, F. Anderl, M. Schiemann, H. Wagner, and D. H. Busch. 2007. A single naive CD8+ T cell precursor can develop into diverse effector and memory subsets. Immunity 27 (6):985-97.

Stier, S., T. Cheng, D. Dombkowski, N. Carlesso, and D. T. Scadden. 2002. Notch1 activation increases hematopoietic stem cell self-renewal in vivo and favors lymphoid over myeloid lineage outcome. Blood 99 (7):2369-78.

Suzuki A. and Ohno S. 2006. The PAR-aPKC systme: lessons in polarity. J.Cell Sci. 119: 979987.

Varnum-Finney, B., L. Xu, C. Brashem-Stein, C. Nourigat, D. Flowers, S. Bakkour, W. S. Pear, and I. D. Bernstein. 2000. Pluripotent, cytokine-dependent, hematopoietic stem cells are immortalized by constitutive Notch1 signaling. Nat Med 6 (11):127881.

Whitman, C.. 1878. The embryology of Clepsine. Q.J. Microsc. Sci. 18: 215.

Wilson, A., D. L. Ardiet, C. Saner, N. Vilain, F. Beermann, M. Aguet, H. R. Macdonald, and O. Zilian. 2007. Normal hemopoiesis and lymphopoiesis in the combined absence of numb and numblike. J Immunol 178 (11):6746-51.

Wirtz-Peitz, F., T. Nishimura, and J. A. Knoblich. 2008. Linking cell cycle to asymmetric division: Aurora-A phosphorylates the Par complex to regulate Numb localization. Cell 135 (1):161-73.

Wu, M., H. Y. Kwon, F. Rattis, J. Blum, C. Zhao, R. Ashkenazi, T. L. Jackson, N. Gaiano, T. Oliver, and T. Reya. 2007. Imaging hematopoietic precursor division in real time. Cell Stem Cell 1 (5):541-54.

Yamashita, Y. M., A. P. Mahowald, J. R. Perlin, and M. T. Fuller. 2007. Asymmetric inheritance of mother versus daughter centrosome in stem cell division. Science 315 (5811):518-21.

Zhong, W., J. N. Feder, M. M. Jiang, L. Y. Jan, and Y. N. Jan. 1996. Asymmetric localization of a mammalian numb homolog during mouse cortical neurogenesis. Neuron 17 (1):43-53. 


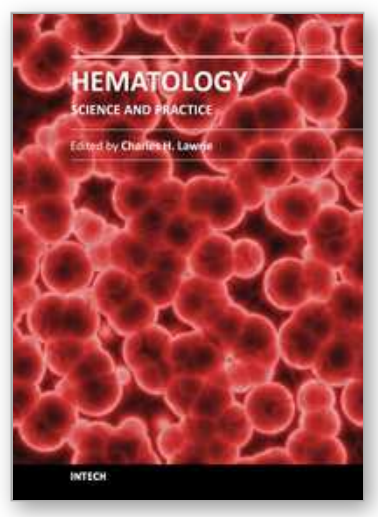

\author{
Hematology - Science and Practice \\ Edited by Dr. Charles Lawrie
}

ISBN 978-953-51-0174-1

Hard cover, 596 pages

Publisher InTech

Published online 02, March, 2012

Published in print edition March, 2012

Hematology encompasses the physiology and pathology of blood and of the blood-forming organs. In common with other areas of medicine, the pace of change in hematology has been breathtaking over recent years.

There are now many treatment options available to the modern hematologist and, happily, a greatly improved outlook for the vast majority of patients with blood disorders and malignancies. Improvements in the clinic reflect, and in many respects are driven by, advances in our scientific understanding of hematological processes under both normal and disease conditions. Hematology - Science and Practice consists of a selection of essays which aim to inform both specialist and non-specialist readers about some of the latest advances in hematology, in both laboratory and clinic.

\title{
How to reference
}

In order to correctly reference this scholarly work, feel free to copy and paste the following:

Daniel Jimenez-Teja, Nadia Martin-Blanco and Matilde Canelles (2012). Asymmetric Division in the ImmunoHematopoietic System, Hematology - Science and Practice, Dr. Charles Lawrie (Ed.), ISBN: 978-953-51-01741, InTech, Available from: http://www.intechopen.com/books/hematology-science-and-practice/asymmetricdivision-in-the-immuno-hematopoietic-system

\section{INTECH}

open science | open minds

\section{InTech Europe}

University Campus STeP Ri

Slavka Krautzeka 83/A

51000 Rijeka, Croatia

Phone: +385 (51) 770447

Fax: +385 (51) 686166

www.intechopen.com

\section{InTech China}

Unit 405, Office Block, Hotel Equatorial Shanghai

No.65, Yan An Road (West), Shanghai, 200040, China

中国上海市延安西路65号上海国际贵都大饭店办公楼405单元

Phone: +86-21-62489820

Fax: $+86-21-62489821$ 
(C) 2012 The Author(s). Licensee IntechOpen. This is an open access article distributed under the terms of the Creative Commons Attribution 3.0 License, which permits unrestricted use, distribution, and reproduction in any medium, provided the original work is properly cited. 